\title{
Nuclear Effects on Oscillation Parameters using Calorimetric method of Neutrino Energy Reconstruction at NOvA experiment
}

\author{
Paramita Deka, ${ }^{a, *}$ Jaydip Singh ${ }^{b}$ and Kalpana Bora ${ }^{a}$ \\ ${ }^{a}$ Department of Physics, \\ Gauhati University, Assam, India \\ ${ }^{b}$ Department of Physics, \\ Lucknow University, Uttar Pradesh, India \\ E-mail: paramitadeka@gauhati.ac.in, jdsingh@fnal.gov, \\ kalpana@gauhati.ac.in
}

\begin{abstract}
Nuclear effects in neutrino scattering interactions are one of the major sources of systematic uncertainties in current and future neutrino-beam oscillation experiments. In this work, we compare the calorimetric and kinematic method of energy reconstruction of the incoming neutrino energy, both at the near and far detector of the NOvA experiment, and study the role of multinucleon (MN) effects, mainly $2 \mathrm{p}-2 \mathrm{~h}$ and RPA on the sensitivity measurement of various neutrino oscillation parameters in the disappearance channel to estimate the size of the systematic error associated with theoretical models. Both short-range and long-range nucleon-nucleon correlations along with Transverse Enhancement are taken into consideration.
\end{abstract}

\footnotetext{
*** The European Physical Society Conference on High Energy Physics (EPS-HEP2021), ***

*** 26-30 July $2021 * * *$

*** Online conference, jointly organized by Universität Hamburg and the research center DESY ***
}

\footnotetext{
${ }^{*}$ Speaker
} 


\section{Introduction}

NUMI Off-Axis $v_{e}$ Appearance Experiment (NOvA) [1] is a 2-detector long-baseline neutrino oscillation experiment which is designed to measure $v_{e}\left(\bar{v}_{e}\right)$ appearance probability and $v_{\mu}\left(\bar{v}_{\mu}\right)$ disappearance probability at Fermilab's NUMI (Neutrinos at the Main Injector) beam. The 290 ton Near Detector (ND) is located $100 \mathrm{~m}$ underground and $1 \mathrm{~km}$ from the production target and $14 \mathrm{kton}$ liquid scintillator Far Detector (FD) is placed at a distance of $810 \mathrm{~km}$ near Ash river, Minnesota.

In this paper, we study the multi-nucleon effects due to long-range Random Phase Approximation (RPA) and 2-particle-2-hole (2p-2h) interactions with Transverse Enhancement (TE) [2] in the sensitivity analysis of neutrino oscillation parameters in $\mathrm{NO} v \mathrm{~A}$. In our recent work we analysed the role of MN without TE using only kinematic method [3]. There are several major processes which produce two (or more) nucleons along with two (or more) holes in the final state - two nucleon correlations in the initial state are referred as the short range correlations (SRC), final state interactions (FSIs) and TE. The transverse enhancement in the QE cross section has been attributed to meson exchange currents (MEC) in a nucleus. If there are no pions in the final state, it is considered as an enhancement of $\mathrm{QE}$ cross section and if one or more pions are created, the process enhances the inelastic cross section.

\section{Simulation and Experimental Details}

Neutrino interactions are simulated using a model configuration of GENIE 3.0.6 [4]. In this custom configuration we use Nieves model [5] for charged current (CC) quasi-elastic (QE) scattering which includes long-range nucleon correlations calculated as per RPA and for $2 \mathrm{p}-2 \mathrm{~h}$ interactions. Another model by Llewellyn-Smith [6] is also considered for QE scattering. The Relativistic Fermi Gas (RFG) model represents the initial nuclear states to account for short-range nucleon correlations. Baryon resonance (RES) and Deep Inelastic Scattering (DIS) effects are simulated using Berger-Sehgal and Bodek-Yang model respectively. GENIE hN semi-classical intranuclear cascade model is used to simulate FSI. For incoming neutrino energy reconstruction, both calorimetric and kinematic method are considered. Calorimetric method [7] is more effective than kinematic method [8] as it is applicable to all types of interactions while kinematic method is exact only for QE interaction as it is based on outgoing lepton kinematics. We have generated 1 million MC events for both ND and FD in the energy range 0-5 GeV using NO $v \mathrm{~A} v_{\mu}$ flux in the Carbon target. The $v_{\mu} \mathrm{CC}$ signal spectra in FD is predicted using the $v_{\mu} \mathrm{CC}$ event spectra in $\mathrm{ND}$, known as extrapolation technique. We have also activated the Transverse Enhancement model (TEM) in GENIE. In sensitivity analysis, the allowed confidence level regions in parameter spaces are obtained using the Feldman-Cousins method [9].

\section{Results and Discussion}

In Figs. 1 and 2, we have shown the comparison of ND and extrapolated FD event distributions with TE as a function of calorimetric and kinematic reconstruction energy for three interactionsQE without RPA, QE with RPA and 2p-2h. The comparison of sensitivity contours using both reconstruction methods is shown in Fig. 3. From Figs. 1 and 2, we observe that the MN effects 
have a significant contribution to the event distribution as a function of energy reconstructed by both the methods. Suppression in the distribution in both ND and FD due to RPA effect is also

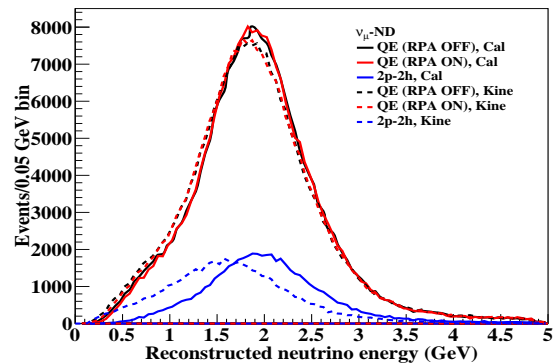

Figure 1: Comparison of ND events as a function of reconstructed $v_{\mu}$ energy using both calorimetric (Cal) and kinematic method (Kine). Events are shown for three different interactions: QE without RPA (black line), QE with RPA (red line), and 2p-2h (blue line).
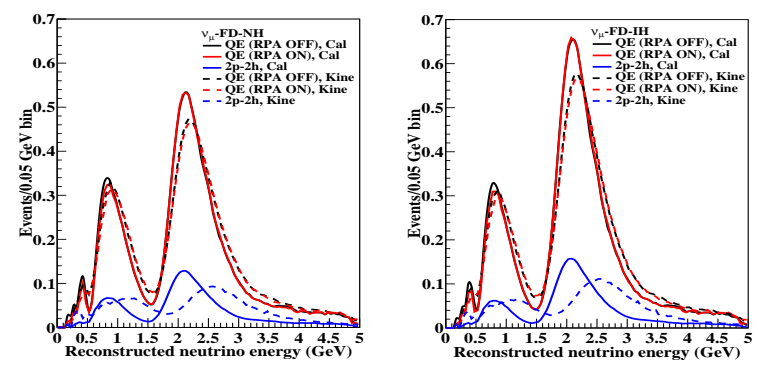

Figure 2: Left and right panel shows comparison of extrapolated FD events as a function of reconstructed $v_{\mu}$ energy for both $\mathrm{NH}$ and IH using both calorimetric and kinematic method. Events are shown for three different interactions: QE without RPA (black line), QE with RPA (red line), and 2p-2h (blue line).

seen. From Fig. 3, we observe that the area of the sensitivity contour deceases with calorimetric method for different interactions. This indicates that the calorimetric method enhances precision in oscillation parameter measurements, as compared to kinematic method.

\section{Summary}

To summarise, in this work, we studied and compared the event distributions at ND and FD, and sensitivity analysis of light neutrino oscillation parameters at disappearance channel of NOvA experiment of Fermilab, USA, using kinematic and calorimetric methods of incoming neutrino energy reconstruction. We also investigated the role of multi-nucleon effects namely $2 \mathrm{p}-2 \mathrm{~h}$ and RPA, with short-range and long range nucleon-nucleon correlations, as well as the TE enhancement contributions. It was found that these contributions have significant effect on sensitivity measurements, and that calorimetric method increases the precision (decreases uncertainties). Hence, these effects should be taken care of and demand a careful inclusion in future measurements at such neutrino oscillation experiments. The results presented here can act as a guidance to include corresponding corrections in the neutrino event generators. 

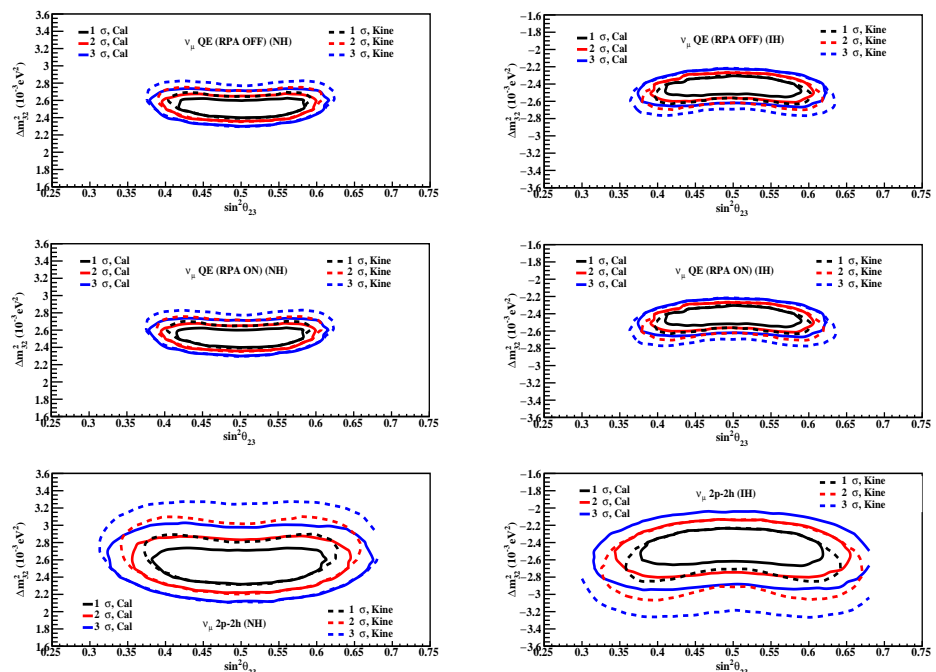

Figure 3: Comparison of $1 \sigma, 2 \sigma$ and $3 \sigma$ contours for $\Delta m_{32}^{2}$ vs $\sin ^{2} \theta_{23}$ for three different interactions QE without RPA (black solid line), QE with RPA suppression (red solid line) and 2p-2h (blue solid line) for NH (left panel) and IH (right panel) for neutrino using both calorimetric and kinematic method.

\section{References}

[1] P. Adamson et al. [NOvA], Phys. Rev. D 93, no.5, 051104 (2016) [arXiv:1601.05037 [hep-ex]].

[2] A. Bodek, H. S. Budd and M. E. Christy, Eur. Phys. J. C 71, 1726 (2011) [arXiv:1106.0340 [hep-ph]].

[3] P. Deka, J. Singh, N. Sarma and K. Bora, [arXiv:2106.16069 [hep-ph]].

[4] C. Andreopoulos, A. Bell, D. Bhattacharya, F. Cavanna, J. Dobson, S. Dytman, H. Gallagher, P. Guzowski, R. Hatcher and P. Kehayias, et al. Nucl. Instrum. Meth. A 614, 87-104 (2010) [arXiv:0905.2517 [hep-ph]].

[5] J. Nieves, J. E. Amaro and M. Valverde, Phys. Rev. C 70, 055503 (2004) [erratum: Phys. Rev. C 72, 019902 (2005)] [arXiv:nucl-th/0408005 [nucl-th]].

[6] C. H. Llewellyn Smith, Phys. Rept. 3, 261-379 (1972).

[7] A. M. Ankowski, P. Coloma, P. Huber, C. Mariani and E. Vagnoni, Phys. Rev. D 92, no.9, 091301 (2015) [arXiv:1507.08561 [hep-ph]].

[8] P. Coloma, P. Huber, C. M. Jen and C. Mariani, Phys. Rev. D 89, no.7, 073015 (2014) [arXiv:1311.4506 [hep-ph]].

[9] G. J. Feldman and R. D. Cousins, Phys. Rev. D 57, 3873-3889 (1998) [arXiv:physics/9711021 [physics.data-an]]. 